\title{
On the Anti-Anthropic Principle (AAP): If there is an Anthropic Principle Exists, There Must be an Anti-Anthropic Principle (AAP) Exists Too
}

\author{
Prasenjit Debnath
}

PhD Student, NIT Agartala, India

\begin{abstract}
According to the anthropic (from Greek word Anthropos, meaning "human") principle, it is a philosophical consideration that the observations of the Universe must have to be compatible with the conscious and sapient life who observes the Universe. Actually, the anthropic principle is kind of a cosmological principle that the theories of the Universe are constraint by the necessity to allow human existence. It explains why the Universe has an age and has some fundamental constants necessary to accommodate conscious and sapient life. Some proponents of the anthropic principles believe that it is unremarkable that the Universe has fundamental constants which exist to fall within the narrow range of thought to be compatible with life on Earth. Anthropic principle has two typesone of them is the strong anthropic principle (SAP) which explains that the anthropic principle holds true for all the cases because the Universe is in some sense compelled to eventually have conscious and sapient life emerge within it. Some critics of SAP argue for weak anthropic principle (WAP) that explains that Universe's ostensible fine tuning is the result of selection bias: there will be living beings capable of observing and reflecting upon fine tuning of the Universe if and only if the Universe is capable of supporting life. Most often this leads to the concept of Multiverse, there are a statistical population of Universes to select from and from which selection bias (our Universe is the only observable Universe because it is compatible with our life) could occur. But it is very psychological way of putting things together in favor of conscious and sapient life. If it is correct, then the reverse also must be true. According to the anthropic principle, if all universal constraints, fundamental constants are there for existence of human beings, then it must be true that sapient lives are also responsible for the Universe the way it is, in other words, sapient lives are the cause of existence of all universal constraints and fundamental constants of the Universe the way these are - if human beings were not there, the Universe would not be that way it is now, implies anti-anthropic principle (AAP). It implies that human beings put controls and impose constraints on the behavior of the Universe to be favorable and observable by them. Human beings might not do that in conscious mind. So, in the conscious mind perspective, the reasoning looks like a joke. Well, joke is a relative word, if you feel this is a joke, then, it is a joke for you. If you feel not, then this is not a joke for you. The same happening can be a joke for one and not joke for the other. According to the Anti-anthropic principle (AAP), human beings put constraints to the Universe either directly or indirectly, either in conscious mind or sub-conscious mind or unconscious mind, how it should behave.
\end{abstract}

Keywords: Anti-anthropic principle (AAP), Strong Anthropic Principle (SAP), Weak Anthropic Principle (WAP), Conscious and Sapient life, Multiverse Statistical Population

\section{Introduction}

According to the Copernican principle, there is nothing special or privileged about Earth or humanity $[1,2]$. Carter draws the same notion in the strong anthropic principle (SAP) [3, 4]. According to Carter, since we live in such a Universe capable of supporting life, then only lifesupporting Universes are possible $[5,6]$. If anthropic principle is the law of relating to human beings or their existence in the Universe [7, 8], the reverse relation is also valid which proves the existence of Anti-anthropic principle (AAP). Because psychology does comparative study with optimization, for us, nanometer is insignificant compared to light years. A human being is nothing compared to the World, the World is nothing compared to the Milky Way, the Milky Way is nothing compared to the observable Universe (having billions of galaxies in it) $[9,10]$. When we optimize the comparison with respect to size, we must detriment ourselves as null and void in comparison with the Universe. But if we can give equal importance to both nanometer and light years, we can conclude that if light year exists in the Universe, so is equally true that nanometer also exists in the Universe. Because relationship is a mutual thing, it implies that if the Universe is there to sustain human race, then, the reverse is also equally true that human race is there to sustain the Universe. If anthropic principle exists, the Anti-anthropic principle (AAP) exists and it is also mutually related and equally valid with respect to anthropic principle and vice versa. If there are infinite constraints of the Universe to make human race viable, there must be equal number of constraints of the human beings to make the Universe the way it is. If you relate me with an amount, then, I also relate you with the same amount. Then, why does not an Anti-anthropic principle (AAP) exist?

\section{Are Accidents Basically Interactions with WIMPS?}

WIMP is weakly interacted massive particle $[11,12]$. It is a hypothetical particle thought to be elementary particle the dark matter made of $[13,14]$. We cannot see them because they do not interact with electromagnetic radiation such as photons (such as sunlight) $[15,16]$. Because they do not have electromagnetic force in them, they do not clump either. Normal ordinary matters like us clumps because of electromagnetic interactions among atoms, the elementary particle, we are made of. Invisible dark matter accounts for $85 \%$ of all matter in the Universe $[17,18]$. It is affecting the motion of the galaxies, bending the path of light, and influencing the structure of the entire Cosmos. Yet we do not know much for certain about its nature. Because they do not clump, they do not bump with ordinary matter like us. But 


\section{International Journal of Science and Research (IJSR) \\ ISSN (Online): 2319-7064}

Index Copernicus Value (2015): 78.96 | Impact Factor (2015): 6.391

because we cannot see them, we can only infer their properties with assumptions based on regular matter (like us, ordinary matter contributes only $4 \%$ of the total matter of the Universe $[19,20])$. Because they interact with gravity as it is already being seen as little bias of WIMPs towards the galaxies compared to the inter galaxies space which is filled up by WIMPs. Inferred from the motions of the galaxies, they interact with ordinary matter with gravity only [21, 22]. Because they contribute $85 \%$ of the total matter of the Universe, they are really massive and produce haloes in the inter galaxies space. It is predicted that 38 WIMPs along with infinite number of neutrinos passing everyday through us with no interaction other than gravitational interaction. These massive heavy particles interact with us with gravity only. Because they are massive, might have little bias on us to do some errors. If the error is negative, we call it is as an accident (negative) and if the error is beneficial for us (positive), we call it as luck or good luck. Nevertheless it is an error. We make error because we are human beings, in a deeper sense, we make error because we interact with something which we do not know, we cannot see. We make error, in most of the cases; it is not our fault at all. At times we are punished for works which are not intentionally done by us. WIMPs interaction made us faulty which leads to an accident either beneficial or malefic for us but nevertheless accident does occur occasionally. Examples of common type of errors made by us, is edging a ball by bat in the game of cricket, error in shot for a goal in soccer football, muscle cramps in the leg while a person in the staircase, a driver hits the car with a tree in nearby roadside. Even intentional errors can be of the influence of WIMPs also. WIMPs can have physical or psychological influence on us or even both influences simultaneously together. Psychological influence can have sudden change of thought process to have an error in judgment although might be momentarily. Physical influence can create an error even it was mentally clear process. Whatever the errors, errors do occur in life by the influences of some unknown fields which interact with our own fields to create errors in judgment either intentionally or unintentionally. WIMPs have a leading role to commit such errors in judgment. From a batter point of view, "I played the ball pretty well; I do not know how it becomes an edge". From a footballer point of view, "I played the ball pretty well; I do not know how it misses the goal post". From a person in the staircase might think, "I put the leg on the right place; I do not know how it become slipping aside". Well, still you can be carefree at worst conditions because of future and past are fixed by time for you, what is allotted for you, can never be blotted for you. How you were going through the passage of time since childhood, you have to go only through the passage of time to the future also.

\section{On The Solar System}

Solar system is highly stable at least for a millions of years $[23,24]$. Sun (also the Milky Way) might have the key part for the stability of the solar system, but it is basically integrated and superimposed interaction of all the planets along with the Sun to make the solar system highly stable. Every planet has its own integral part to make the solar system highly stable. So, all the planets along with Sun are responsible for the stability of Earth, thus, all the planets effecting Earth more or less for attaining and keeping stability. From the anthropic principle point of view, the whole solar system exists at the right place so that sapient life can be viable in the Earth. From the Anti-anthropic principle point of view, Human beings are responsible for the solar system the way it is. All the planets are at the knife edge accuracy so that sapient life can be flourished on the Earth. On psychological point of view, life is itself on the knife edge also and everyone is perfect at their own place. It is only the place from where you are looking at - either positive or negative. The same number you can read as six from one side and can be read nine from the opposite side and both readings are correct from their respective side.

\section{Conclusion}

From sapient life point of view, the anthropic principle is absolutely correct. If the anthropic principle is correct, then there exists an anti-anthropic principle (AAP) which has an opposite reasoning compared to the anthropic principle is also absolutely correct because if human race is related to the Universe qualitatively and quantitatively, then the Universe is also related to us by the same way, qualitatively and quantitatively. If light year exists in the Universe, a nanometer also exists in the Universe. Both has unique right place to use them both, but a comparative study will lead us to a joke only. Microscopic and macroscopic both are perfect at their own right place. It needs accurate optimization for using right thing at the right place at the right time. Thus, anthropic principle and anti-anthropic principle (AAP) are equally related to each other, if one exists, the other exists too. It looks odd to compare human race with the Universe, but the odd itself is also a relative word, in that sense nothing is odd, everything is right at its own place.

\section{Acknowledgment}

I am cordially grateful to Dr. Aparna Nath, Associate Professor and my PhD Guide, The department of Physics, National Institute of Technology, Agartala, India, for the epitome of inspiration and motivation to write this particular paper with perfection and accuracy. I am extremely thankful to her for all possible help she made to write this paper. Also I am thankful to The Department of Physics of National Institute Of Technology Agartala (NIT Agartala) for proper conduct and coordination.

\section{References}

[1] Barrow, John D., Tipler, Frank J. "The Anthropic Cosmological Principle", Oxford University Press, ISBN 978-19-282147-8, LCCN 87028148, 1988.

[2] Cirkovic, M.M. "On The First Anthropic Argument in Astrobiology", Earth, Moon, and Planets. 91 (4):243254, doi:10.1023 /A:1026266630823, 2002.

[3] Cirkovic, M.M. "The Anthropic Principle and the Duration of Cosmological Past", Astronomical and Astrophysical Transactions. 23(6): 567-597, 2004.

[4] http://en.m.wikipedia.org/wiki/Anthropic principle

[5] Roger Penrose, "Cycles of Time", Vintage Books, London, pp. 50-56.

\section{Volume 6 Issue 1, January 2017 www.ijsr.net}




\section{International Journal of Science and Research (IJSR) \\ ISSN (Online): 2319-7064 \\ Index Copernicus Value (2015): 78.96 | Impact Factor (2015): 6.391}

[6] Stephen Hawking, "A Briefer History of Time”, Bantam Books, London, pp. 1-49.

[7] Stephen Hawking, "Black holes and Baby Universes and other essays", Bantam Press, London 2013, ISBN 978-0-553-40663-4

[8] Stephen Hawking, "The Grand Design", Bantam Books, London 2011

[9] Stephen Hawking, "A Brief History of Time", Bantam Books, London 2011, pp. 156-157. ISBN-978-0-55310953-5

[10] Stephen Hawking, "The Universe in a Nutshell", Bantam Press, London 2013, pp. 58-61, 63, 82-85, 90-94, 99, 196. ISBN 0-553-80202-X

[11] Stephen Hawking, "The Beginning of Time", A Lecture.

[12] Stephen Hawking, "Stephen Hawking's Universe: Strange Stuff Explained", PBS site on imaginary time.

[13] Gerald D. Mahan, "Many-Particle Physics", Third Edition, Springer, 2000

[14] Uno Ingard, K "Fundamental of Waves \& oscillations", Cambridge University Press. P. 38, ISBN-0-521-33957XOxford: The British Academy, 1999

[15]A. Zee, "Quantum Field Theory in a Nutshell", Princeton University Press, 2003

[16] Storrs McCall, "A Model of the Universe", Oxford: Clarendon Press, 1994

[17] Craig Callender, "Time, Reality and Experience", Cambridge, UK: Cambridge University Press.

[18]Craig Callender, "Thermodynamic Asymmetry in Time", The Stanford Encyclopedia of Philosophy (Spring 2002 Edition)

[19] Storrs McCall, "A Model of the Universe", Oxford: Clarendon Press, 1994

[20] Robin Le Poidevin and Murray McBeath, "The Philosophy of Time" Oxford: Oxford University Press, 1993

[21] Whitrow, G., "The Natural Philosophy of Time". Oxford: Oxford University Press, 1961. (2nd edn., 1980.)

[22] Smart, J. J. C., "Problems of Space and Time". London: Macmillan, 1964

[23] Stephen Hawking, "A stubbornly persistent illusionThe essential scientific works of Albert Einstein", Running Press Book Publishers, Philadelphia, London 2011.

[24] William L.Craig, "Time and the Metaphysics of Relativity", Dordrecht: Kluwer Academic Publisher, 2001

\section{Author Profile}

Prasenjit Debnath, born in Agartala, Tripura, India on $15^{\text {th }}$ of March 1979. He is pursuing PhD in the Department of Physics in National Institute of Technology Agartala (NIT Agartala), India. 